\title{
Eficacia de la terapia neural en pacientes con dolor cervical o lumbar, del servicio de medicina física y rehabilitación de un centro hospitalario, 2016 Effectiveness of neural therapy in patients with neck pain or low back pain, of rehabilitation unit, 2016
}

\author{
Diana Marcela Villamizar Olarte ${ }^{1}$, Daicy Rojas de Rangel ${ }^{1}$
}

\section{RESUMEN}

Objetivos: 1) Evaluar la eficacia de la terapia neural (TN) con procaína al 1\% comparada con placebo. 2) Comparar la TN segmentaria con y sin tratamiento de campos interferentes, en pacientes con dolor cervical o lumbar de la consulta externa del servicio de Medicina Física y Rehabilitación en un centro hospitalario. Materiales y métodos: Ensayo clínico controlado, aleatorizado, simple ciego, en pacientes con cervicalgia o lumbalgia, de causa mecánica, sin fibromialgia, sin alergia a anestésicos locales, ni realización simultánea de terapia física. Intervención: 3 sesiones de orden semanal, con procaína 1\% o placebo (SSN 0,9\%) bajo la técnica de pápulas intradérmicas, iniciando con terapia segmentaria y derivando a manejo de campos interferentes según el fenómeno segmentario presentado. Revaloración a los 7, 14 y 21 días, con medición de EVA y consumo de medicamentos. Además, escala de funcionalidad al inicio y final de tratamiento.

Resultados: Sesenta y seis pacientes, $83 \%$ mujeres, mediana de 41 años, $47 \%$ intervenidos con placebo, $53 \%$ con procaína. EVA inicial 5,85 (DE: $\pm 1,72$ ) y final 2,16 (DE: $\pm 1,72$ ), con mejoría significativa a favor de TN con procaína en todas las mediciones ( $\mathrm{p}<0,001)$. A nivel funcional se encontró mejoría a favor de TN con procaína ( $p<0,001)$, del grupo de lumbalgia $(p<0,001)$ y de los tratados con campos interferentes $(p<0,001)$. No hubo diferencia en el consumo de fármacos entre los grupos. Efectos secundarios escasos, sin repercusiones y resolución espontánea.

Conclusión: La TN demostró ser eficaz y segura en el tratamiento del dolor cervical y lumbar, además de mejorar la funcionalidad de los pacientes con lumbalgia y los tratados en campos interferentes.

Palabras clave: terapia neural, dolor, cervicalgia, lumbalgia.

DOI: http://dx.doi.org/10.28957/rcmfr.v27n1a2

\section{ABSTRACT}

Objective: 1) To evaluate the effectiveness of neural therapy (NT) with procaine $1 \%$ compared to placebo. 2) To compare segmental NT with and without treatment interference fields in patients with neck pain or low back pain, of physical medicine and Rehabilitation Unit.

Materials and methods: Controlled clinical trial, randomized, single-blind, in patients with neck pain or low back pain, mechanical cause, without fibromyalgia, without allergy to local anesthetics, or simultaneous physical therapy. Intervention: 3 sessions weekly with procaine 1\% or placebo (SSN0.9\%) under the technique of intradermal papules, starting with segmental therapy and drifting to management interference fields according segmental phenomenon presented. Evaluation at 0, 7, 14 and 21 days, measuring AVS and consumption of medicines. Additionally, functionality scale was applied at the beginning and at the end of treatment.

Results: 66 patients, $83 \%$ female, median 41 years, $47 \%$ treated with placebo, $53 \%$ with procaine. Initial vas $5.85(\mathrm{SD} \pm 1.72)$ and final 2.16 $(\mathrm{SD} \pm 1.72)$, with significant improvement in favor of NT with procaine in all measurements ( $p<0.001)$. Functionally improvement for NT with procaine $(p<0.001)$, low back pain group $(p<0.001)$ and treated with interference fields $(p<0.001)$. There was no difference in consumption medicines between groups. Few adverse events were presented and it had spontaneous resolution.

Conclusion: NT proved to be effective and safe in the treatment of neck pain and low back pain, and improve the functionality of patients with low back pain and treated with interfering fields.

Keywords: pain, neural therapy, neck pain, low back pain.

DOI: http://dx.doi.org/10.28957/rcmfr.v27n1a2

Autores:

${ }^{1}$ Médica y cirujana.

Especialista en

Medicina Física y

Rehabilitación.

Instituto Autónomo Hospital

Universitario de los

Andes. Mérida,

Venezuela

Recibido:

enero 25 de 2017

Aceptado:

mayo 3 de 2017

Correspondencia:

marchkdanllet@gmail.com

Conflictos de interés:

Ninguno 


\section{INTRODUCCIÓN}

El dolor es una de las afecciones principales del ser humano moderno. Es por ello que podemos considerarlo un problema importante de salud pública, debido a las alteraciones funcionales y psicológicas que puede producir en los individuos, además de los costos que genera para los sistemas de salud ${ }^{1}$. Dentro del dolor musculoesquelético, la cervicalgia y la lumbalgia adoptan un papel protagónico, dada su frecuencia dentro de la población y como causa de algún grado de discapacidad en personas menores de 45 años $^{2}$. Se calcula entre un $50 \%$ y un 70 a $80 \%$ de probabilidad de que un individuo en algún momento de su vida presente un dolor cervical o lumbar, respectivamente, siendo este último el más prevalente con un 18 a $21 \%$ en la población general $3,4,5$.

Si bien existen diferentes modalidades terapéuticas como el tratamiento médico, agentes físicos, masajes, manipulaciones, tracciones y ejercicios terapéuticos ${ }^{2,6}$, el incremento de las cifras de dolor y las mejorías parciales, causantes en los pacientes de desesperanza, consultas repetitivas, frustraciones personales y laborales, plantean la necesidad de proponer estrategias de intervención alternativas y/o complementarias, con el objetivo de modificar positivamente la calidad de vida de los pacientes.

La terapia neural (TN) es en la actualidad una técnica dentro de las medicinas alternativas y/o complementarias que podría ser utilizada para el alivio del dolor. Es una técnica que utiliza pequeñas cantidades de anestésico local, idealmente procaína al 0,5 a 1\%, depositada en piel o en zonas profundas del cuerpo, la cual actúa sobre el sistema nervioso neurovegetativo. Al realizar la aplicación de la terapia se logra con la introducción de la aguja estimular fibras nerviosas somáticas que producen un freno presináptico y con el anestésico local desactivar fibras nerviosas vegetativas, cerrando la compuerta del dolor propuesta por Melzack y Wall en $1965^{7}$ y rompiendo el círculo vicioso: dolor, tensión muscular, isquemia y dolor, favoreciendo su restablecimiento fisiológico.
Es preciso conocer que la TN contempla 5 tipos de aplicaciones según Huneke ${ }^{8,9}: 1$ ) sitio de dolor o «locus dolendi», 2) segmento según HEAD, 3) sistema nervioso autónomo (ganglios autónomos simpáticos), 4) intravasal y 5) campos interferentes. Se define este último como cualquier estímulo irritativo crónico que permanece en la memoria del sistema neurovegetativo como por ejemplo, cicatrices o procesos cro ' nicos, que pueden causar cambios patológicos a distancia en un momento determinado ${ }^{10}$.

Es importante identificar las diferentes reacciones que se producen después de la aplicación de TN segmentaria y de campos interferentes reconocidos como fenómenos de la terapia neural (de Hopfer y Huneke, respectivamente), esto con el objetivo de definir el plan terapeútico más apropiado para el paciente ${ }^{11}$ (tabla 1).

Así pues, los médicos que manejan dolor pueden intervenir sobre el segmento y campo interferente presente, pudiendo mejorar la salud de sus pacientes, priorizando una determinada actuación, sin olvidar que lo ideal es un abordaje de tipo integral ${ }^{12}$.

En cualquier caso, aunque el uso de esta técnica requiere un proceso de formación definido, la aplicación de la terapia neural en sí es extraordinariamente sencilla. Sin embargo, es poco conocida, en parte por la escasez de trabajos publicados en revistas de impacto científico sobre su eficacia a nivel de dolor y más específicamente de dolor cervical y lumbar. Por esta razón, creemos necesario evaluar el aporte de la medicina alternativa al manejo de estas enfermedades como lo plantea la Organización Mundial de la Salud en su estrategia sobre medicina tradicional 2014$2023^{13}$. Los objetivos de este estudio consistieron en 1) evaluar la eficacia de la TN con procaína al 1\% comparada con placebo, y 2) comparar la TN segmentaria con y sin tratamiento de campos interferentes, en pacientes con dolor cervical o lumbar, de la consulta externa del Servicio de Medicina Física y 
Tabla 1. Descripción de los fenómenos de la terapia neural según el tipo de intervención. (Adaptado de Nissnick M. La terapia neural en odontología. 2.a ed. Venezuela. Biotecnoquímica; 2013)

\begin{tabular}{|c|c|}
\hline Segmentaria (según Hopfer) & Campos interferentes (según Huneke) \\
\hline $\begin{array}{l}\text { Fenónemo del segmento: La aplicación de un anestésico local } \\
\text { en áreas dolorosas o puntos de presión que resulta en una } \\
\text { mejoría de las molestias y desaparición de las mismas. Con } \\
\text { estas observaciones aisladas (Schleich, Spiess, Leriche, } \\
\text { Huneke) se desarrolló el concepto de la terapia de } \\
\text { segmento. } \\
\text { Fenómeno de reacción: Consiste en un empeoramiento del } \\
\text { cuadro durante } 2 \text { o } 3 \text { días después de un tratamiento } \\
\text { segmental con TN. Posterior a este, las molestias se reducen } \\
\text { otra vez al punto de partida. El fenómeno de reacción es, en } \\
\text { el marco de diagnóstico segmental, el indicador para una } \\
\text { enfermedad producida por un campo interferente. Al } \\
\text { fenómeno de reacción se le debe, entonces, seguir con la } \\
\text { búsqueda del campo interferente, ya que el persistir con el } \\
\text { tratamiento segmental no llevará al éxito. }\end{array}$ & $\begin{array}{l}\text { Fenónemo en segundos: este fenómeno se caracteriza por: } \\
\text { 1) Desaparición en un } 100 \% \text { de todas las molestias a } \\
\text { distancia producidas por el campo interferente, hasta } \\
\text { donde la anatomía lo permita. } \\
\text { 2) La total liberación de los síntomas tiene que mantenerse } \\
\text { por lo menos } 8 \text { horas si el campo interferente está en la } \\
\text { cavidad bucal y } 20 \text { horas si se halla en cualquier otra parte } \\
\text { del organismo. } \\
\text { 3) Si aparecen los síntomas y aplicamos nuevamente el } \\
\text { tratamiento neural en el mismo campo interferente } \\
\text { observamos que la liberación total de los síntomas tiene } \\
\text { una mayor duración que en la aplicación anterior. } \\
\text { Fenómeno inverso: Reacción que consiste en un empeo- } \\
\text { ramiento de los síntomas distante al sitio de aplicación del } \\
\text { tratamiento neuralterapéutico seguido de una mejoría que } \\
\text { tiene una duración mínima igual a la observada en el } \\
\text { fenómeno en segundos. } \\
\text { Fenómeno tardío: se caracteriza por: } \\
\text { 1) La reacción de la mejoría se presenta después de media } \\
\text { hora o de una hora después de la aplicación del trata- } \\
\text { miento neuralterapéutico. } \\
\text { 2) La liberación de los síntomas a distancia debe tener una } \\
\text { duración de } 10 \text { minutos si es la cavidad bucal y } 20 \text { minutos } \\
\text { el resto del organismo. } \\
\text { 3) El fenómeno tardío es el más frecuentemente observado. } \\
\text { La reacción tardía puede significar que en el órgano blanco } \\
\text { se encuentra establecido un proceso crónico, o que los } \\
\text { mecanismos de reacción están disminuidos por empan- } \\
\text { tanamiento mesenquímico. }\end{array}$ \\
\hline
\end{tabular}

\section{Rehabilitación del Instituto Autónomo Hospital Universitario de los Andes, entre enero y junio de 2016.}

\section{MATERIALES Y MÉTODOS}

Se realizó un estudio experimental, tipo ensayo clínico, controlado, aleatorizado, simple ciego, desarrollado en el área de consulta externa del Servicio de Medicina Física y Rehabilitación del Instituto Autónomo Hospital Universitario de los Andes, entre enero y junio de 2016.

\section{Criterios de inclusión}

a. Pacientes de 18 o más años. b. Ambos géneros.

c. Dolor cervical o lumbar de causa mecánica.

d. Pacientes sintomáticos.

e. Comorbilidades compensadas.

f. Firma de consentimiento informado.

\section{Criterios de exclusión}

a. No firma de consentimiento informado.

b. Dolor cervical o lumbar, con criterios quirúrgicos o de causa no mecánica.

c. Fibromialgia.

d. Realización de terapia física al momento de iniciar el estudio.

e. Alergia a anestésicos locales.

f. Enfermedad psiquiátrica o crónica de base descompensada.

Eficacia de la terapia neural en pacientes con dolor cervical o lumbar, del servicio de medicina física y rehabilitación de un centro hospitalario, 2016 
Rev Col Med Fis Rehab 2017;27(1):25-37 - http://revistacmfr.org DOI: http://dx.doi.org/10.28957/rcmfr.v27n1a2

\section{Procedimiento}

Se aplicó un protocolo de una sesión semanal hasta completar tres sesiones, con una valoración cada 7 días posterior a la última aplicación (días $0,7,14$ y 21).

Una vez fue confirmada la elegibilidad del paciente con base en los criterios de inclusión y exclusión, se procedió a la explicación verbal del procedimiento, dando a conocer en qué consistía y sus posibles efectos secundarios. El paciente no tuvo conocimiento si la intervención en él se realizaría con placebo (SSN 0,9\%) o procaína al 1\%. Con ello se realizó la firma del consentimiento informado y se diligenció la ficha de recolección de datos, la cual comprendía información sociodemográfica, clínica y funcional (tabla 2). Posteriormente se asignó al azar (a través de una aplicación Android para celular) a uno de los 2 grupos: placebo o procaína y se procedió a realizar la primera aplicación del tratamiento.

El procedimiento inicia con medidas de asepsia, antisepsia y delimitación de los segmentos a tratar según las zonas de HEAD, para posteriormente realizar a nivel paravertebral en los territo- rios segmentarios relevantes, la técnica de pápulas intradérmicas con depósito de aproximadamente $0,2-0,4$ cc de SSN $0,9 \%$ o procaína al $1 \%$. El número de pápulas osciló entre 8 y 12 , de acuerdo con los segmentos a tratar (figura 1).

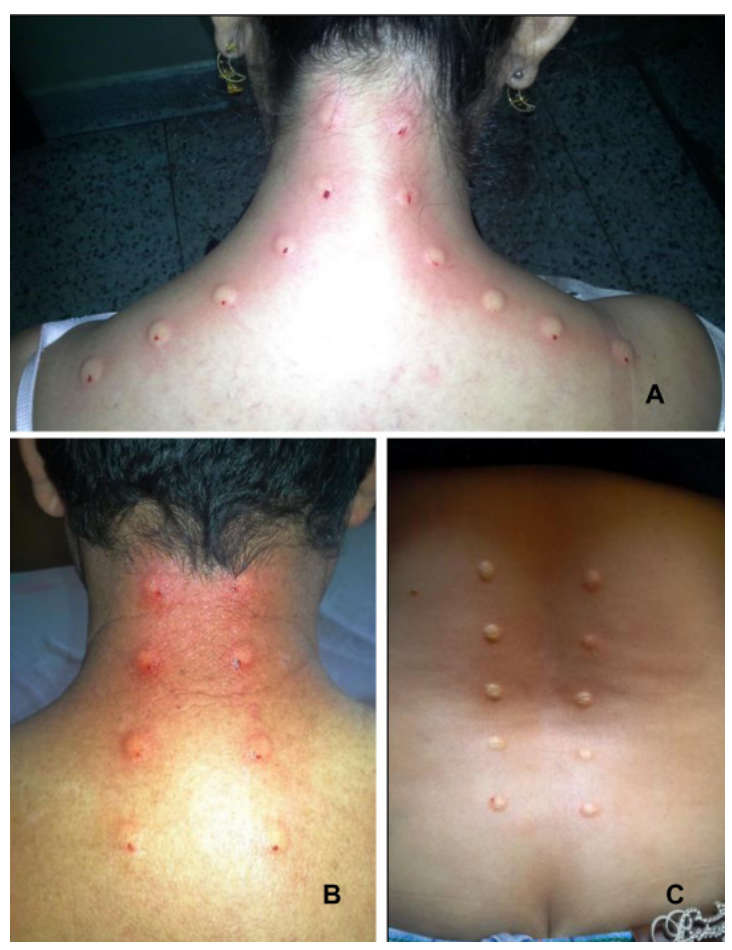

Figura 1. Terapia neural segmentaria cervical (Ay B) y lumbar (C).

Tabla 2. Sistema de variables

\begin{tabular}{l|l}
\hline & Clínicas \\
Sociodemográficas & Ebicación del dolor \\
Edad & Tiología \\
Género & IMC de evolución \\
Estado civil & Terapia física previa \\
Procedencia & Campos interferentes presentes \\
Escolaridad & EVA \\
Tipo de trabajo & Consumo de medicamentos (tipo, frecuencia)* \\
-SP & \\
-BPP & Funcionales \\
-Carga & Índice de discapacidad cervical / Cuestionario de \\
-SCE & discapacidad lumbar de Oswestry** \\
& Mínima, moderada, intensa, discapacidad, \\
& limitación máxima \\
\hline
\end{tabular}

SP: Sedestación prolongada, BPP: Bipedestación prolongada, SCE: Sin características específicas, IMC: Indice de masa corporal, EVA: Escala visual análoga.

* La evolución de la ingesta se determinó con base en los cambios de su frecuencia de consumo en aumentó, disminuyó, eliminó o igual.

** La mejoría, empeoramiento o igualdad se determinó con base en el cambio de nivel funcional. 
El paciente fue citado a los 7 (semana 1$)$ y 14 días (semana 2) a partir de la primera intervención terapéutica, para revalorar el nivel de dolor a través de la escala visual análoga (EVA) y registrar los cambios en el consumo de medicamentos. Además, se registró el fenómeno segmentario presentado, el cual determinó si en esta segunda y tercera intervención (semana 1 y 2) continuaba con TN segmentaria o si requería el tratamiento de campos interferentes (figura 2). En caso de no presentar algún fenómeno o presentar empeoramiento, el paciente era intervenido con la última modalidad de terapia utilizada (figura 3).

Una última valoración se realizó a los 21 días (semana 3), para registrar el nivel final de dolor, cambio en el consumo de medicamentos y escala de funcionalidad realizada al ini-
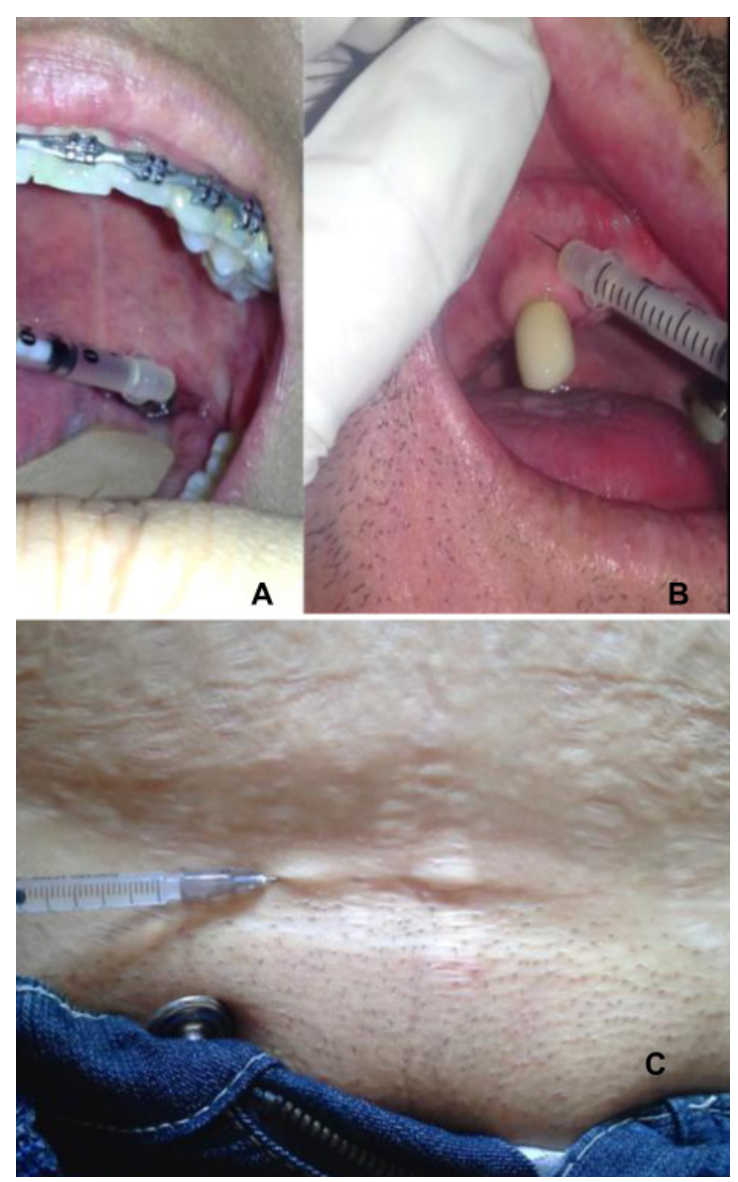

Figura 2. Terapia neural en campos interferentes. Polos amigdalinos (A), dentario (B) y cicatrices (C) cio de la intervención terapéutica (Índice de discapacidad cervical/Cuestionario de discapacidad lumbar de Oswestry, según correspondía) ${ }^{14,15}$, considerando el cambio de nivel como punto de mejoría, empeoramiento o igualdad.

\section{Control de sesgos}

Si bien al desarrollar un estudio simple ciego, existe el riesgo de que los sujetos sean influenciados por la interacción con el investigador al conocer este qué tipo de terapia recibiría el paciente (sesgo cognitivo), este se controló con la asignación de la terapia después de la entrevista inicial y diligenciamiento de la ficha de recolección de datos, donde se expusieron las características, beneficios y efectos secundarios de la terapia, asumiendo en un primer momento que todos recibirían la terapia verdadera.

El sesgo de memoria que pudo estar presente en el seguimiento, ya que se programó de forma semanal, se controló pidiendo al paciente que registrara por escrito la evolución y cambios con respecto al dolor y aparición de cualquier sensación o síntoma local y sistémico, al menos durante los tres primeros días posteriores a la intervención terapéutica.

\section{Consideraciones éticas}

La realización del presente trabajo se adecuó a las recomendaciones para la investigación biomédica de la Declaración de Helsinki de la Asociación Médica Mundial

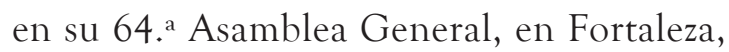
Brasil, en octubre de $2013{ }^{16}$ y lo contemplado en el Código de Deontología Médica de la Federación Médica Nacional del 20 de marzo de 1985, en su Título V, Capítulo 4, referente a la investigación en seres humanos ${ }^{17}$. Además, se tuvo en cuenta la gran trayectoria de la terapia neural, la cual lleva más de 70 años en nuestro medio demostrando ser una terapia segura y eficaz. 
Rev Col Med Fis Rehab 2017;27(1):25-37 - http://revistacmfr.org

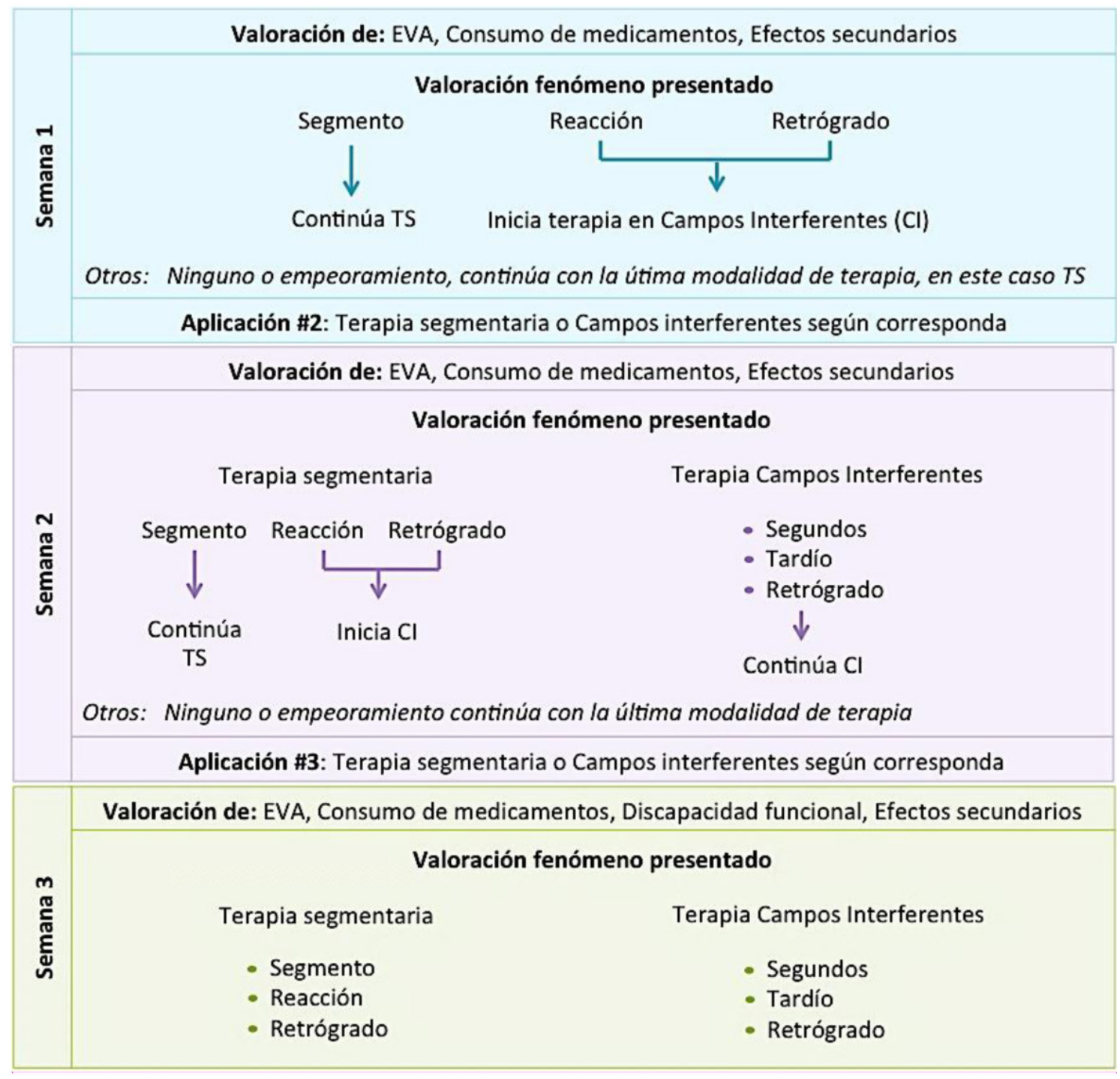

Figura 3. Protocolo de trabajo a partir de la $1 .^{a}$ semana de seguimiento, donde se determinaba la modalidad de terapia neural con la cual se realizaría la 2. ${ }^{a}$ y $3 .^{a}$ aplicación. TS: Terapia segmentaria. CI: Campos interferentes.

\section{Procesamiento y análisis estadístico}

Se tabuló la información recolectada a través de la ficha de recolección de datos en el programa Microsoft EXCEL 2011. Posteriormente fue procesada en el programa SPSS versión 20.0 para windows, realizando un análisis descriptivo con números y porcentaje, y t-student para muestras independientes. Las relaciones entre variables se establecieron con chi-cuadrado. Se utilizó Anova de medidas repetidas para determinar la evolución del dolor, primero en el grupo total y luego en el grupo con procaína.

\section{RESULTADOS}

El número de pacientes intervenidos fue de 66 , el $47 \%$ con placebo y el $53 \%$ con procaína al $1 \%$. El $83 \%$ era del género femenino y el $17 \%$ del género masculino, con una edad entre los 20 y 69 años, mediana de 41 años. El 52\% eran casados, seguidos por el $30 \%$ solteros. El $82 \%$ era procedente del área urbana. En su nivel educativo el $46 \%$ era bachiller, el $26 \%$ profesional, el $18 \%$ técnico y el $10 \%$ contaba solo con estudio primarios. El tipo de trabajo predominante fue bipedestación prolongada en un 
$30 \%$, sin características específicas en un $27 \%$, sedestación prolongada $26 \%$ y finalmente actividades de carga en un $17 \%$.

De los pacientes evaluados el $53 \%$ presentaba dolor cervical y el $47 \%$ dolor lumbar. Respecto al tiempo de evolución, el $85 \%$ presentaba un dolor crónico (mayor a 3 meses), siendo en el 36,4\% de 1 a 5 años de duración. En la medida de índice de masa corporal (IMC) el 64\% presentaba sobrepeso y solo el $3 \%$ algún grado de obesidad.

En cuanto a la etiología se destaca la presencia de alteraciones musculares dadas por contracturas o síndrome doloroso miofascial en un $78,8 \%$ y con proporciones similares entre sí, discopatía degenerativa, problemas posturales y artrosis (tabla 3). El 36,4\% había realizado terapia física previa al inicio de la intervención. En la determinación de campos interferentes se encontró un rango de 0 a 10, en donde el $73 \%$ tenía entre 2 y 4 campos interferentes presentes al inicio de la terapia, en su mayoría a nivel dental y procesos crónicos.

La media de nivel de EVA al inicio fue de 5,85 (DE: $\pm 1,72$ ), siendo para el grupo placebo de 4,93 (DE: $\pm 1,43$ ) y de procaína 6,62 (DE: $\pm 1,57$ ). Al final del tratamiento sus niveles fue- ron de 3,21 (DE: $\pm 1,72$ ) y 1,29 (DE: $\pm 1,16)$ para placebo y procaína respectivamente, con una media de 2,16 (DE: $\pm 1,72$ ) para el grupo en general. Al realizar Anova para medidas repetidas se encontró significancia estadística a favor del tratamiento con procaína en todas las evaluaciones del seguimiento $(p<0,001)$, presentando una pendiente mayor durante la primera y segunda aplicación (figura 4).

El nivel de discapacidad funcional más frecuente de inicio fue la moderada con $47 \%$, seguida por mínima $(24,5 \%)$, intensa $(21,2 \%)$, discapacidad (4,5\%) y máxima (3\%), siendo al final de la intervención los niveles mínimo y moderado los más predominantes con un 47 y $43,9 \%$ respectivamente. Al estudiar la mejoría, empeoramiento o igualdad entre el inicio y final de los grupos placebo vs. procaína, se evidenció una mejoría en el nivel de discapacidad funcional a favor de este último grupo con una $\mathrm{p}<0,001$ (figura 5).

Dentro del consumo de medicamentos para control del dolor se encontró el consumo de AINES, paracetamol, tramadol, relajantes musculares y neuromoduladores, siendo el consumo de AINES el más frecuente con un 68,2\% (tabla 4). Para evaluar la progresión de ingesta de los mismos, se consideró su aumento,

Tabla 3. Descripción de la etiología de los pacientes a quienes se les aplicó terapia neural.

\begin{tabular}{ccccc}
\hline \multirow{2}{*}{ Etiología } & \multicolumn{2}{c}{ Frecuencia } & \multirow{2}{*}{ Porcentaje } \\
\cline { 2 - 4 } & Cervical & Lumbar & Total & \\
\hline Muscular & 30 & 22 & 52 & 78,8 \\
Discopatía degenerativa & 10 & 14 & 24 & 36,4 \\
Postural & 14 & 10 & 24 & 36,4 \\
Artrosis & 13 & 9 & 22 & 33,3 \\
Rectificación & 12 & 0 & 12 & 18,2 \\
Hiperlordosis & 0 & 4 & 4 & 6,1 \\
Escoliosis & 0 & 2 & 2 & 3 \\
Espondilolistesis & 0 & 2 & 2 & 3 \\
\hline
\end{tabular}

Tabla 4. Descripción del tipo de medicamentos consumidos por los pacientes al inicio del tratamiento con terapia neural.

\begin{tabular}{ccc}
\hline Medicamento & Frecuencia & Porcentaje \\
\hline AINES & 45 & 68,2 \\
Relajante muscular & 17 & 25,8 \\
Paracetamol & 16 & 24,2 \\
Tramadol & 11 & 16,7 \\
Neuromodulador & 11 & 16,7 \\
\hline
\end{tabular}

Eficacia de la terapia neural en pacientes con dolor cervical o lumbar, del servicio de medicina física y rehabilitación de un centro hospitalario, 2016 


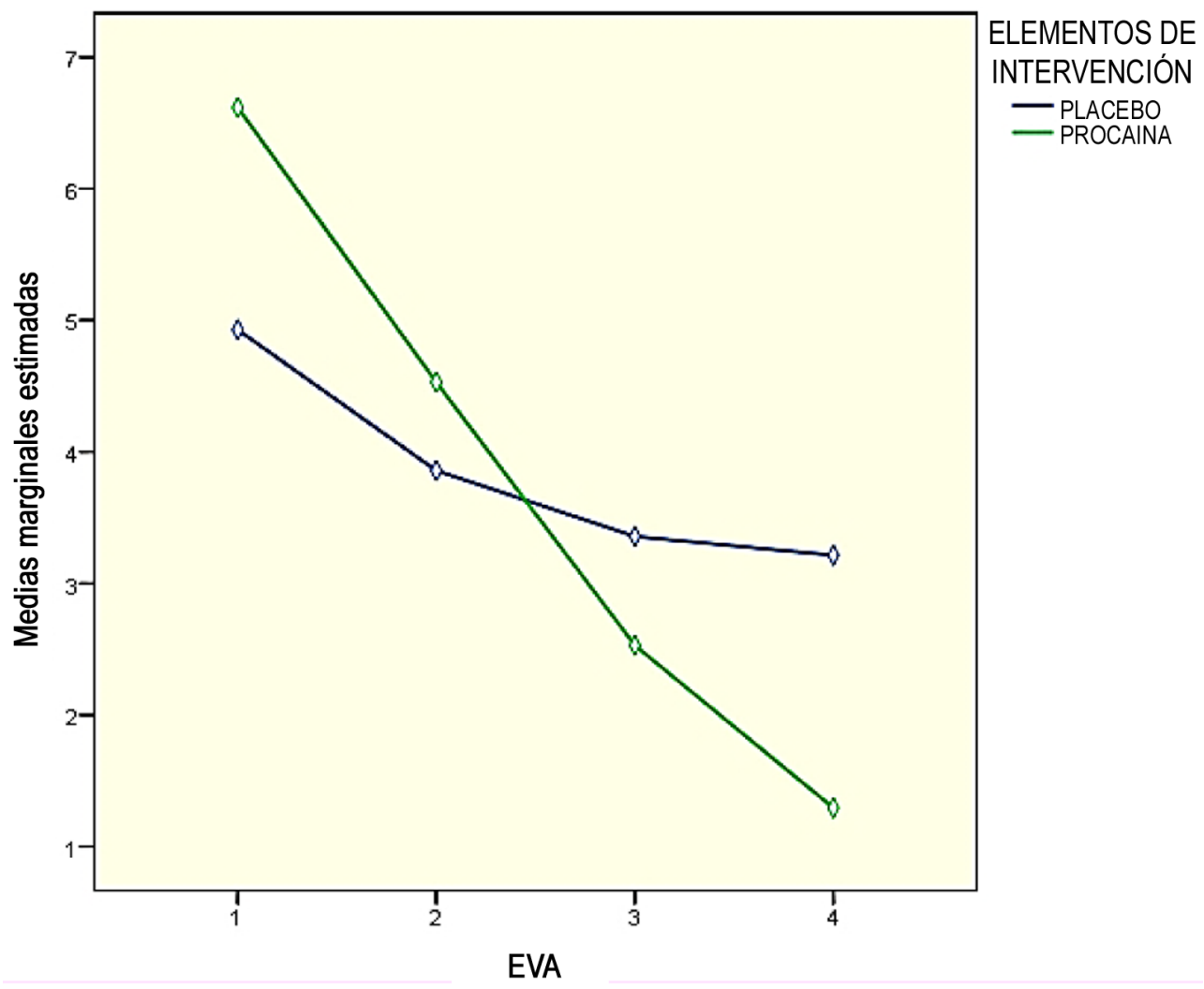

Figura 4. Nivel de EVA durante todo el seguimiento en los grupos de placebo vs. procaína

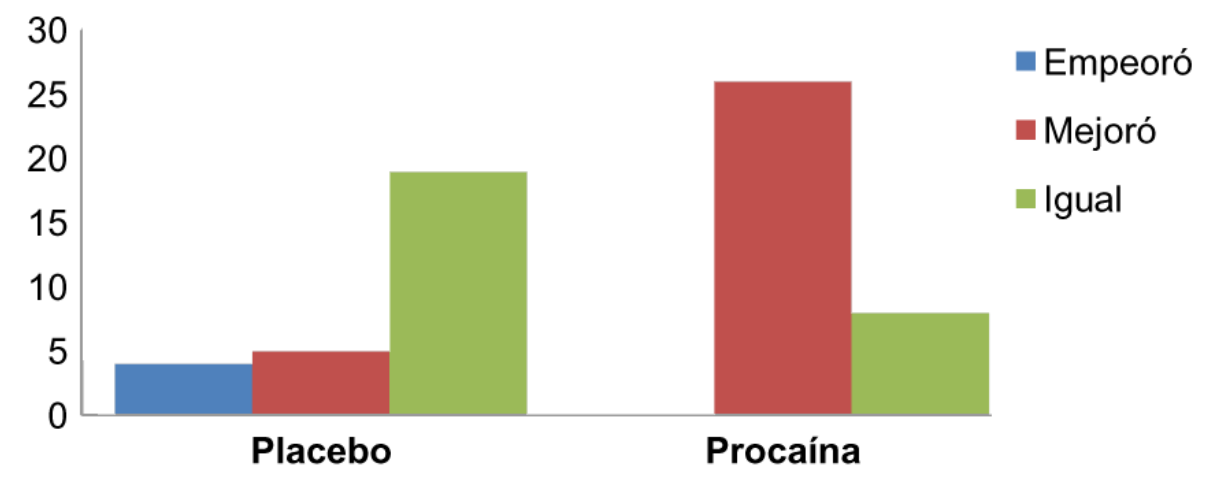

Figura 5. Conclusión de nivel de discapacidad funcional al final del tratamiento entre el grupo de placebo y el de procaína $(\mathrm{p}<0,001)$

disminución, eliminación o igualdad durante cada semana, encontrándose un comportamiento similar entre los grupos.

Al tomar el grupo intervenido con procaína y estudiar el comportamiento de los pacientes con cervicalgia vs. lumbalgia, encontramos que para los niveles de EVA no hubo diferencias estadísticamente significativas entre estos dos grupos, mostrando un comportamiento similar, coincidiendo hacia el final del seguimiento en la tercera aplicación (figura 6). Caso contrario, al analizar la discapacidad funcional donde se obtuvo una mejoría estadísticamente significativa a favor del grupo con dolor lumbar ( $<<0,001)$, comparado con el grupo de dolor cervical $(\mathrm{p}>0,06)$. 


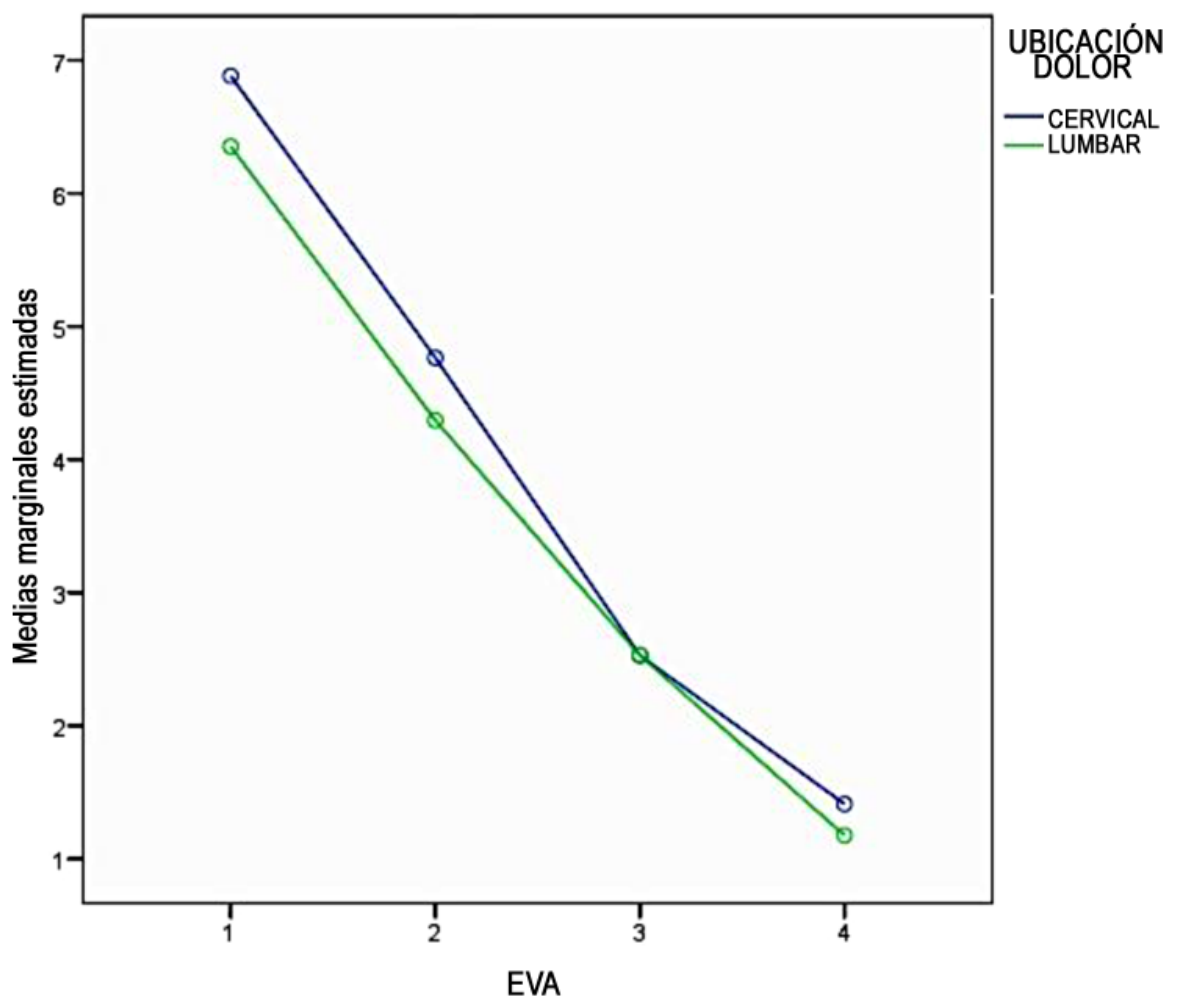

Figura 6. Nivel de EVA durante todo el seguimiento en los grupos de cervical vs. lumbar intervenidos con procaína

Al comparar en el grupo de procaína la modalidad de terapia (segmentaria o campos interferentes), se encontraron diferencias para las mediciones de EVA tanto de terapia segmentaria como de campos interferentes desde el inicio hasta la tercera aplicación. Sin embargo, en la valoración final su nivel de EVA fue muy similar para ambos grupos, sin encontrarse diferencia estadísticamente significativa al terminar el tratamiento (figura 7). Con respecto al nivel de discapacidad final, se encontraron diferencias estadísticamente significativas $(\mathrm{p}<0,001)$ a favor de mejoría en el grupo tratado en la modalidad de campos interferentes y a favor de mantenerse igual o sin cambios en aquellos que recibieron terapia segmentaria. Adicionalmente, se pudo apreciar que el fenómeno de la TN segmentaria más frecuente fue el segmentario, seguido por el de reacción, y en el caso de la TN en campos interferentes el fenómeno más común fue el tardío.
Se pudo registrar como efectos secundarios de terapia neural con procaína: un caso de ardor moderado en el sitio de aplicación el día de la intervención, un caso de calor en la zona intervenida, un paciente con cefalea de una hora posterior a primera intervención y un caso de mareo en los cinco minutos posteriores a la aplicación de la terapia; todos ellos sin repercusión en el tiempo y con resolución espontánea.

\section{DISCUSIÓN}

Esta investigación se da bajo la escasez de estudios científicos que evalúan la eficacia de la terapia neural comparada con placebo, así como su efectividad en grupos de dolor cervical versus lumbar, siendo aún más deficientes los estudios de la terapia neural segmentaria comparada con el tratamiento de campos interferentes. A pesar de ello, se acepta y están en proceso de desarrollo investigaciones para 


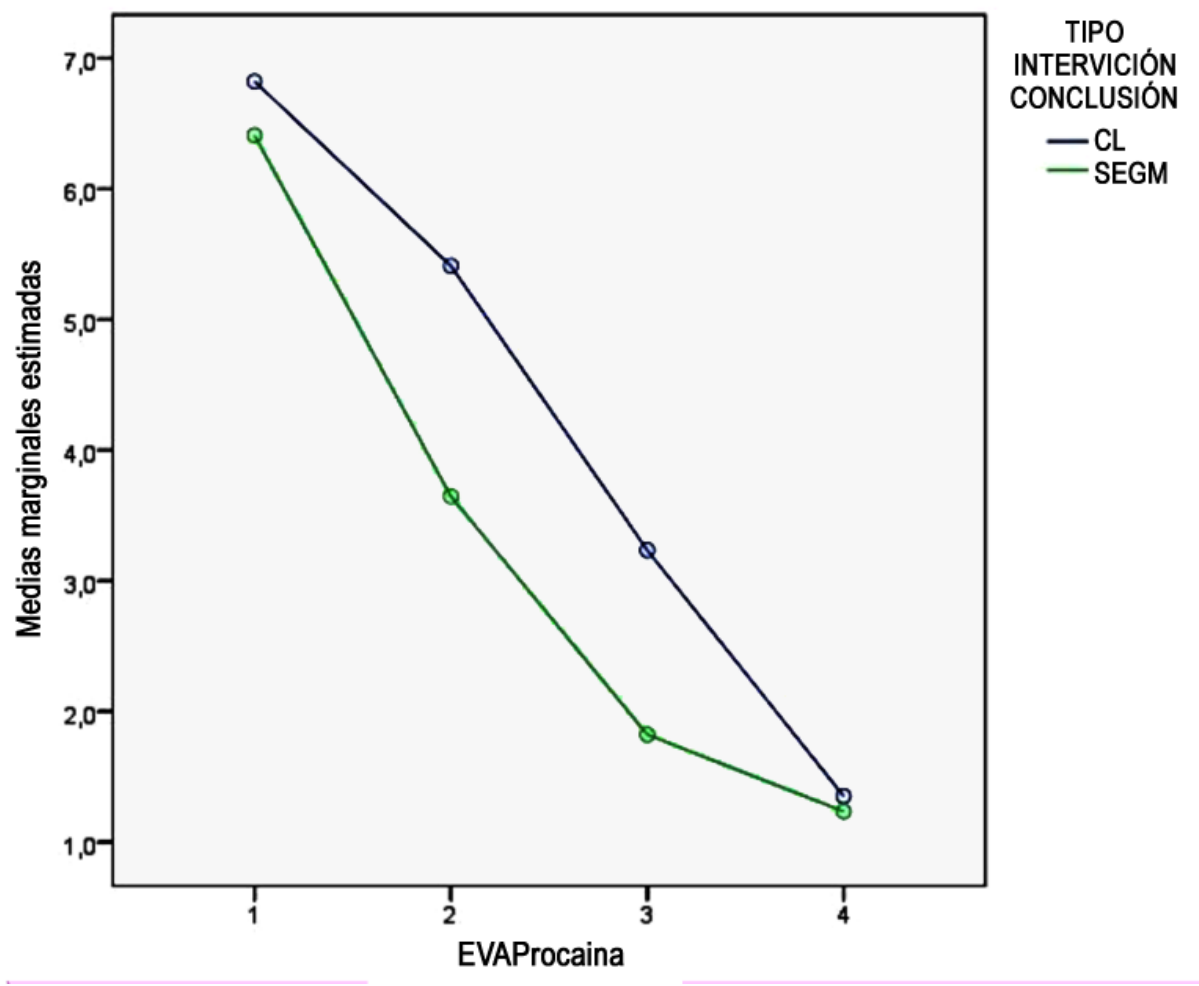

Figura 7. Nivel de EVA durante todo el seguimiento para los pacientes intervenidos con procaína en la modalidad de terapia segmentaria vs. campos interferentes

comprobar la eficacia de este tipo de terapia no solo en el tratamiento de dolor sino en el de múltiples condiciones que pueden jugar un papel protagónico en el bienestar y desarrollo del ser humano ${ }^{18}$.

Se pudo comprobar la eficacia de la TN con procaína al 1\% comparada con placebo en las mediciones de dolor cervical y lumbar a través de EVA, reduciendo su valor en cada una de las aplicaciones para llegar a un nivel final de 1,29 (DE: $\pm 1,16)$ con un protocolo de tres aplicaciones de orden semanal. Estos resultados coinciden con varios estudios experimentales donde se logró una disminución significativa del dolor durante las primeras semanas de tratamiento $19,20,21$.

Era importante determinar los cambios en el nivel funcional del grupo general, teniendo en cuenta que contenían, a su vez, dos subgrupos dados por la ubicación del dolor: cervical y lumbar. Se logró evaluar este comportamiento de manera conjunta, eligiendo como escalas de medición el índice de discapacidad cervical y el cuestionario de discapacidad de Oswestry, ya que ambas escalas contienen 10 componentes equiparables entre sí, permitiendo evaluar la condición de estos dos grupos de forma agrupada, comprobando que la TN con procaína mejora el nivel funcional al final del tratamiento.

Llama la atención la mejoría en los niveles de dolor y discapacidad funcional a favor del grupo de TN con procaína, a pesar de que el consumo de fármacos fue similiar en ambos grupos. Al analizar esta situación, se observó que las personas que disminuyeron o eliminaron el tratamiento farmacológico en el grupo placebo no lo realizaron por mejoría del dolor sino por fallas en la disponibilidad del medicamento, lo cual es reflejo de la situación que actualmente está atravesando el país. 
Si bien la literatura habla de la lumbalgia como principal motivo de consulta con respecto a la cervicalgia, la proporción de casos de dolor lumbar en este estudio fue inferior con respecto al dolor cervical. Esto probablemente por la noción de aplicabilidad de la TN más en la zona cervical que la lumbar, lo que llevó a un incremento en la referencia de este tipo de casos.

Tomando el grupo intervenido con procaína, se observó que la TN es efectiva tanto a nivel cervical como lumbar en la mejoría del dolor, sin llegar a ser superior en alguno de los dos grupos. Caso contrario, a nivel funcional, donde se observó mejoría en los pacientes con lumbalgia; no así en pacientes con cervicalgia, lo cual puede ser debido a múltiples factores que influirían a nivel cervical para que esta limitación se perpetúe, tales como el estado emocional, estrés y otros factores psicosociales; además de la existencia de protocolos de prevención ya establecidos, dirigidos principalmente a la lumbalgia y no a la cervicalgia. No se encontraron estudios de TN que evaluaran la funcionalidad a nivel cervical para correlacionar estos hallazgos. Aunque sí existen investigaciones a nivel lumbar, que favorecen el uso de la terapia neural en la mejoría de la funcionalidad 22,23 .

Uno de los principales interrogantes en la práctica de la $\mathrm{TN}$ es con respeto a la TN segmentaria y de campos interferentes. Se observó que la terapia segmentaria presenta una pendiente mayor en la disminución del dolor en las primeras aplicaciones; no así en el tratamiento de campos interferentes. Sin embargo, al final del seguimiento ambas modalidades lograron resultados similares, lo cual indica una adecuada interpretación de los fenómenos segmentarios, para poder intervenir eficazmente a quien requiera el tratamiento adicional de campos interferentes.

Finalmente, según los resultados de este estudio, la TN presenta efectos secundarios escasos, reafirmando su seguridad en la práctica regular de la consulta externa.

\section{CONCLUSIONES}

La terapia neural con procaína al $1 \%$, comparada con placebo, demostró ser eficaz en el tratamiento del dolor tanto cervical como lumbar; a la vez que mejora la funcionalidad de los pacientes con lumbalgia.

La terapia neural segmentaria y la terapia neural en campos interferentes fueron igualmente efectivas en el alivio del dolor bajo el protocolo propuesto. Cabe resaltar que esta última modalidad mejora el nivel funcional de los pacientes intervenidos bajo esta técnica.

La terapia neural demostró ser una herramienta segura, eficaz y sencilla en el manejo del paciente con dolor cervical y lumbar.

\section{RECOMENDACIONES}

Es conveniente el seguimiento a mediano y largo plazo, para evaluar los efectos de la terapia neural en el tiempo.

En estudios futuros se debe ampliar la muestra de pacientes con dolor agudo para lograr realizar comparaciones con el dolor crónico y determinar si la terapia neural es igualmente eficaz en los dos grupos o existen diferencias entre ellos.

Se recomienda el uso complementario de terapia neural con procaína al $1 \%$ en el manejo del dolor cervical y lumbar, interviniendo de inicio los campos interferentes identificados, bajo un esquema mínimo de una sesión semanal por al menos 3 semanas.

Continuar con investigaciones relacionadas al uso de la terapia neural en otros tipos de patologías y síndromes dolorosos.

\section{AGRADECIMIENTOS}

Agradecemos especialmente a todos los pacientes que nos permitieron desarrollar esta in-

Eficacia de la terapia neural en pacientes con dolor cervical o lumbar, del servicio de medicina física y rehabilitación de un centro hospitalario, 2016 
vestigación y al apoyo del Servicio de Medicina Física y Rehabilitación del Instituto Autónomo Hospital Universitario de los Andes, en la aceptación y derivación de los mismos al personal médico encargado de la aplicación de la terapia neural, considerando este tipo de medicina alternativa una opción en el manejo del dolor de los pacientes atendidos en la consulta fisiátrica.

\section{CONTRIBUCIÓN DE LOS AUTORES}

Diana Marcela Villamizar Olarte: investigadora principal y desarrolladora del estudio en todas sus fases.

Daicy Rojas de Rangel: consultante en el diseño de la investigación y colaboradora en la captación de pacientes para el estudio.

\section{RESPONSABILIDADES ÉTICAS}

\section{Protección de personas y animales}

Los autores declaran que para esta investigación no se han realizado experimentos en seres humanos ni en animales.

\section{Confidencialidad de los datos}

Los autores declaran que en este artículo no aparecen datos de pacientes.

\section{Derecho a la privacidad y consentimiento informado}

Los autores declaran que en este artículo no aparecen datos de pacientes. 


\section{REFERENCIAS}

1. Waxman R, Tennant A, y Helliwell P. Community survey of factors associated with consultation for low back pain. BMJ.1998;3 17(7172):1564-7.

2. Maher, CG. Effective physical treatment for chronic low back pain. Orthop Clin North Am. 2004;35(1):57-64.

3. Raspe H, Matthis C, Croft P, O'Neill T. Variation in back pain between countries: the example of Britain and Germany. Spine. 2004;29(9):1017-21.

4. Hoy D, Brooks P, Blyth F, et al. The Epidemiology of low back pain. Best Pract Res Clin Rheumatol. 2010;24:769-81.

5. Romero C, Prieto M, Calle C. Las cervicalgias en la consulta de atención primaria. FMC. 2012; 19(9):521-8.

6. Mannion AF, Mu"ntener M, Taimela S, Dvorak, J. Comparison of three active therapies for chronic low back pain: results of a randomized clinical trial with one-year follow-up. Rheumatology. 2001;40(7):772-8.

7. Melzack R, Wall PD. Pain mechanisms: A new theory. Science. 1965;150(3699):971-9.

8. Nissnick M. La terapia neural en odontología. 2. a ed. Venezuela. Biotecnoquímica; 2013.

9. Fisher. Terapia neural según Huneke. Fundamentos, técnica, aplicación práctica. México. Hipócrates Verlag Stuttgart. 2000: 88-95; 100,1 .

10. Fischer L. Neuraltherapie. Schweiz Z Ganzheitsmed. 2010;22:114-6.

11. Nissnick M. La terapia neural en odontología. 2. a ed. Venezuela. Biotecnoquímica; 2013.

12. Dickenson AH. Gate control theory of pain stands the test of time. Br J Anaesth. 2002; 88(6):755-77.

13. Organización Mundial de la Salud. Estrategia de la OMS sobre medicina tradicional 2014-2023. Ginebra 2013.

14. Andrade JA, Delgado A, Almécija R. Validación de una versión española del índice de discapacidad cervical. Med Clin. 2008; 130(3):85-9.
15. Alcántara-Bumbiedro S, Flórez-García MT, Echávarri-Pérez C, García-Pérez F. Escala de incapacidad por dolor lumbar de Oswestry. Rehabilitación. 2006;40(3):150-8.

16. Asociación Médica Mundial. Declaración de Helsinki de la Asociación Médica Mundial. Principios éticos para las investigaciones médicas en seres humanos. 64. ${ }^{\text {a }}$ Asamblea General, Fortaleza, Brasil. WMA [Internet]. 2013 [citado 16 abr. 2016]. Disponible en: http://www.wma.net/es/30publications/ 10policies/b3/

17. Federación Médica Venezolana. Código de Deontología Médica. LXXVI Reunión extraordinaria de la Asamblea de la Federación Médica Venezolana [Internet]. 1985 [citado 16 Abr 2016]. Disponible en: http:/ /www.gobiernoenlinea.ve/home/ lesgislación.dot

18. Becke H. Possibilities of neural therapy in internal medicine. Z Gesamte Inn Med. 1988;43(22):643-5.

19. Chiriboga C, Rodríguez V, Proaño P, Salinas F. Dolor cervical y Terapia Neural. XIV Jornadas Médicas Hospital Alcivar. Ecuador 2006 [Citado 17 Abr 2016]. Disponible en: http://www.terapianeural.com/component/ content/article? $\mathrm{id}=135$ : dolor

20. Lóriz O, Raya A, Pérez D. Estudio de intervención sobre el dolor subagudo y crónico en atención primaria: una aproximación a la efectividad de la terapia neural. Aten Primaria. 2011;43(11):604-10.

21. Egli S, Pfister M, Ludin SM, Puente de la Vega K, Busato A, Fischer L. Long-term results of therapeutic local anesthesia (neural therapy) in 280 referred refractory chronic pain patients. BMC Complement Altern Med. 2015;15:200-9.

22. Medrano R, Valera H, Torre M, Mendoza R, Acosta Y. Resultados de la aplicación de la terapia neural en la lumbalgia inespecífica. Archivo Médico de Camagüey. 2011; 15(1):1-13.

23. Atalay NS, Sahin F, Atalay A, Akkaya N. Comparison of efficacy of neural therapy and physical therapy in chronic low back pain. Afr J Tradit Complement Altern Med. 2013;10(3):431-5. 